\title{
REVIEW \\ The effectiveness of 22 commonly administered physiotherapy interventions for people with spinal cord injury: a systematic review
}

\author{
LA Harvey, JV Glinsky and JL Bowden
}

\begin{abstract}
Study design: A systematic review of randomised controlled trials. Objectives: To determine the effectiveness of 22 commonly administered physiotherapy interventions for people with spinal cord injuries (SCls).

Methods: The inclusion criteria contained 22 pairs of key interventions and outcomes. Each intervention and outcome was considered independently such that 22 brief reviews were conducted and summarised in this one paper. The interventions included hand, wheelchair, seated mobility, fitness, strength and gait training, as well as electrical stimulation, passive movements, stretch, cycling, exercise and transcutaneous electrical nerve stimulation (TENS). Interventions were compared with no or sham treatment, usual care or another physiotherapy intervention. The primary outcome for each review was one of the following: seated mobility, wheelchair mobility, hand function, gait, voluntary strength, joint mobility, fitness and pain. Data were extracted to determine mean between-group differences or risk ratios (95\% confidence interval). Where possible, results were pooled in meta-analyses and the strength of evidence rated using Grading of Recommendations Assessment, Development and Evaluation.

Results: Thirty-eight randomised controlled trials met the inclusion criteria and were relevant to 15 of the brief reviews. The following four interventions were clearly effective: fitness, hand and wheelchair training as well as TENS; however, the strength of evidence was not high for any of these interventions. None of the other interventions were clearly effective.

Conclusion: There is initial evidence to support four physiotherapy interventions, but there is still a long way to go to put a strong evidence base to the range of physiotherapy interventions commonly used to manage people with $\mathrm{SCl}$.

Spinal Cord (2016) 54, 914-923; doi:10.1038/sc.2016.95; published online 28 June 2016
\end{abstract}

\section{INTRODUCTION}

There are numerous systematic reviews, literature reviews and clinical practice guidelines summarising the effectiveness of different physiotherapy interventions for people with spinal cord injuries (SCIs). ${ }^{1-12}$ However, most include non-randomised studies that are highly vulnerable to bias. There are some high-quality Cochrane Systematic Reviews, but they often include interventions not typically administered by physiotherapists or include people with different types of neurological conditions. They are also very detailed, which can limit their accessibility. ${ }^{11,13-15}$ We wanted to provide an unbiased but very accessible summary of the evidence underpinning physiotherapy practice as part of a larger project devoted to adding 'evidence tips' to the physiotherapy module of www.elearnSCI.org. For this reason, we conducted 22 brief reviews that were restricted to randomised controlled trials. The reviews are 'brief' because each examines the effectiveness of an intervention on one primary outcome. The results of each review are pooled in this one paper to provide an overall summary of the evidence about the effectiveness of a range of different but commonly administered physiotherapy interventions.

\section{MATERIALS AND METHODS}

Twenty-two brief reviews were conducted. Each brief review looked at the effectiveness of an intervention on one primary outcome (Table 1), and each intervention/outcome pair was considered independently. The interventions and outcomes were selected a priori and reflected those of most interest to physiotherapists, and those described within www.elearnSCI.org. The list of interventions and outcomes is not exhaustive, and unlike a typical systematic review we did not look at all the possible effects of any single intervention.

\section{IDENTIFICATION AND SELECTION OF STUDIES}

The following electronic databases were searched for publications up until December 2015: Medline, CINAHL, Embase, the Cochrane Central register of controlled trials and the Physiotherapy Evidence Database (PEDro). A search strategy for randomised controlled trials ${ }^{7}$ was used along with the following terms: parapleg\$, quadripl\$, tetrapleg\$, wheelchair\$ and spinal cord. This search strategy was adjusted for each database.

Two reviewers screened publications by title and abstracts against the inclusion criteria. Full copies of potentially eligible trials were

John Walsh Centre for Rehabilitation Research, Kolling Institute, Sydney Medical School/Northern, Faculty of Medicine, University of Sydney, St Leonards, New South Wales, Australia

Correspondence: Professor L Harvey, John Walsh Centre for Rehabilitation, Kolling Institute, Sydney Medical School/Northern, C/O Royal North Shore Hospital, Pacific Highway, St Leonards, New South Wales 2065, Australia.

E-mail: I.harvey@usyd.edu.au

Received 13 January 2016; revised 24 March 2016; accepted 2 May 2016; published online 28 June 2016 
Table 1 The intervention, comparison, outcome, number of trials, conclusions and GRADE rating for each of the 22 comparisons.

\begin{tabular}{|c|c|c|c|c|c|}
\hline Intervention & Comparison ${ }^{\mathrm{a}}$ & Outcome & No of trials & Conclusions & GRADE rating \\
\hline Seated mobility training & No or sham intervention & Seated mobility & $2^{28,41}$ & Inconclusive & - \\
\hline Wheelchair mobility training & No or sham intervention & Wheelchair mobility & $1^{51}$ & Effective & Very low \\
\hline Hand training & No or sham intervention & Hand function & $1^{25}$ & Effective & Very low \\
\hline Hand training+ES & No or sham intervention & Hand function & $1^{18}$ & - & - \\
\hline Overground gait training & No or sham intervention & Gait & - & - & - \\
\hline Overground gait training+ES & No or sham intervention & Gait & - & - & - \\
\hline BWSTT & No or sham intervention & Gait & - & - & - \\
\hline BWSTT+ES & No or sham intervention & Gait & - & - & - \\
\hline BWSTT & Overground gait training & Gait & $7^{24,33,34,43,47,52,55}$ & Inconclusive & - \\
\hline BWSTT+ES & Overground gait training & Gait & $1^{50}$ & Inconclusive & - \\
\hline Robotic gait training & No or sham intervention & Gait & $1^{17}$ & - & - \\
\hline Robotic gait training & Overground gait training & Gait & $3^{23,43,53}$ & Inconclusive & - \\
\hline Stretch & No or sham intervention & Joint range of motion & $5^{26,}, 30,32,37,38$ & Ineffective & Moderate \\
\hline Passive movements & No or sham intervention & Joint range of motion & $2^{22,39}$ & Inconclusive & - \\
\hline Strength training for non-paralysed muscles & No or sham intervention & Voluntary strength & $2^{42,48}$ & Inconclusive & - \\
\hline Strength training for partially paralysed muscles & No or sham intervention & Voluntary strength & $1^{35}$ & Inconclusive & - \\
\hline Strength training+ES for partially paralysed muscles & No or sham intervention & Voluntary strength & $1^{40}$ & Inconclusive & - \\
\hline ES for partially paralysed muscles & No or sham intervention & Voluntary strength & $5^{20,21,36,44,49}$ & Inconclusive & - \\
\hline Fitness training & No or sham intervention & Fitness & $4^{42,45,46,54}$ & Effective & Moderate \\
\hline Cycling with ES & No or sham intervention & Fitness & - & - & - \\
\hline General exercise & No or sham intervention & Pain & $3^{31,42,48}$ & Inconclusive & - \\
\hline TENS & No or sham intervention & Pain & $2^{27,29}$ & Effective & Moderate \\
\hline
\end{tabular}

Abbreviations: BWSTT, bodyweight supported treadmill training; ES, electrical stimulation; GRADE, grading of recommendations assessment, development and evaluation; TENS, transcutaneous electrical nerve stimulation

aSometimes included usual care provided usual care was also provided to the intervention group.

retrieved and again screened for eligibility. Any disagreements between the two reviewers were resolved by a third independent reviewer.

\section{INCLUSION CRITERIA}

\section{The participants}

The participants of interest were people with SCI. Trials involving people with conditions other than SCI were only included if at least $75 \%$ of the participants had sustained a SCI. Trials involving predominantly children were excluded.

\section{The interventions}

The interventions of interest were seated mobility training, wheelchair mobility training, electrical stimulation (ES), hand training (with and without ES), overground gait training (with and without ES), bodyweight supported treadmill training (BWSTT, with and without ES), robotic gait training, strength training (for non-paralysed and partially paralysed muscles, with and without ES), stretch, passive movements, fitness training, cycling with ES, general exercise and transcutaneous electrical nerve stimulation (TENS). Trials were only included if the intervention was administered on more than one occasion.

\section{The comparison}

Trials were included if they compared the interventions of interest with no intervention or a sham intervention. Robotic and BWSTT were also compared with overground walking. Trials that included cointerventions or usual care were included if the co-interventions or usual care were administered to both groups to make it possible to determine the added benefit of the intervention of interest.

\section{The outcomes}

One outcome was pre-determined for each brief review (Table 1). For example, the primary outcome for the review about BWSTT was gait, and the primary outcome for the review about stretch was joint range of motion. The primary outcome for each review was one of the following: seated mobility, wheelchair mobility, hand function, gait, voluntary strength, joint mobility, fitness and pain. If a trial included two or more measures of the same outcome (for example, Walking Index for Spinal Cord Injury (WISCI) and $10 \mathrm{~m}$ walk test to reflect gait), then one measure was chosen, which best reflected the outcome of interest. This measure was chosen without looking at the results of the trial and using a decision rule that prioritised measures according to whether they were:

1. Identified by the authors as the primary outcome (either in the paper or in the trial registry)

2. Easily interpretable by clinicians

3. Reported in sufficient detail to determine mean between-group differences or risk ratios and corresponding 95\% confidence intervals.

\section{Types of studies}

Only randomised controlled trials written in English were included. Cross-over trials were included provided allocation to the treatment schedule was randomised. Trials with more than two parallel comparisons were included provided two of the comparisons met the inclusion criteria. If trials were published more than once or interim analyses were published prior to the completion of the trial, then the most recent or most relevant publication was retrieved.

\section{DATA EXTRACTION}

One reviewer extracted study characteristics and two reviewers extracted outcome data from the included studies onto a standardised Excel spreadsheet. Data from only one time period were used for each trial and reflected the first time period after the intervention ceased. For example, if a trial examined a 6-week gait training programme and 


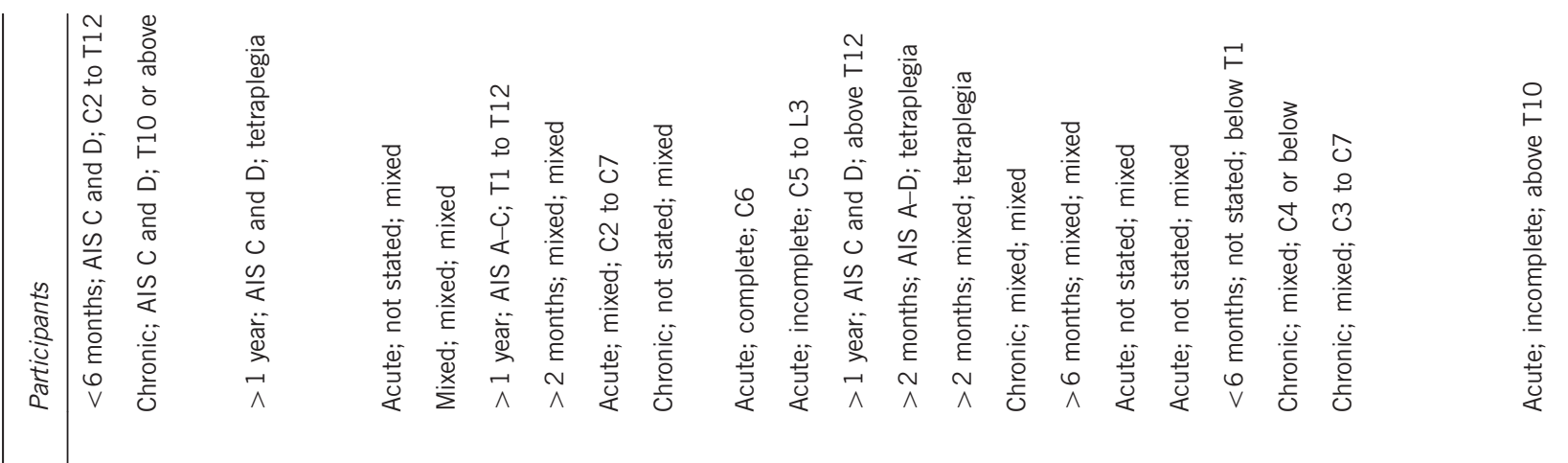

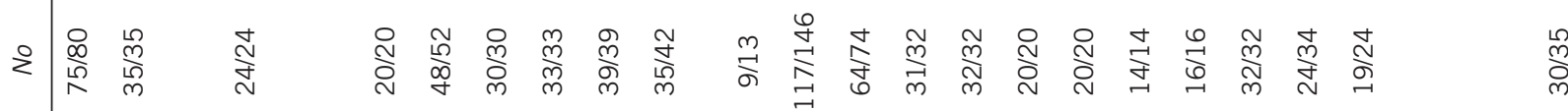

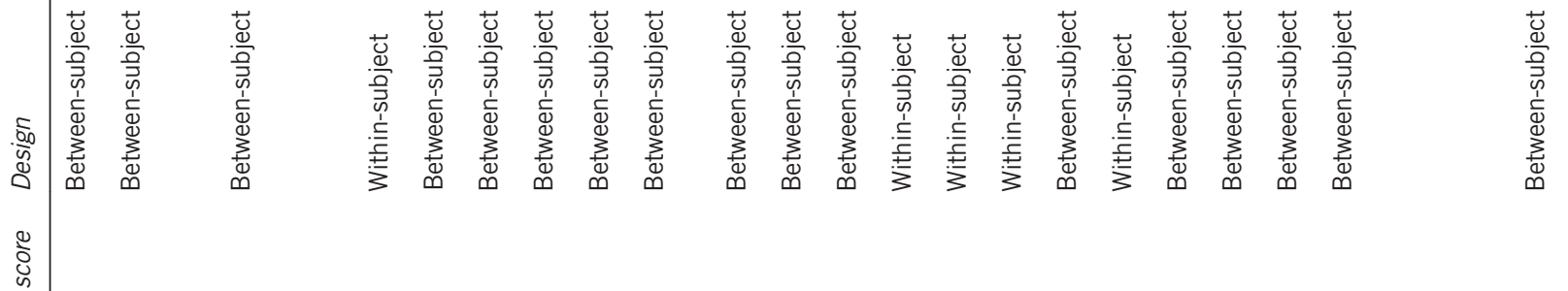

育

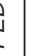

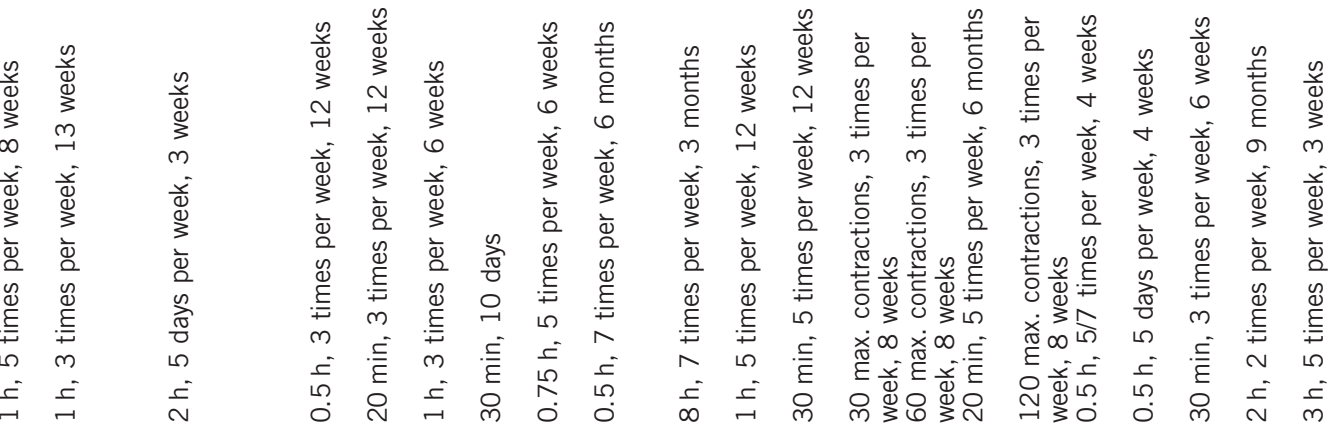

$\sqrt{2}$

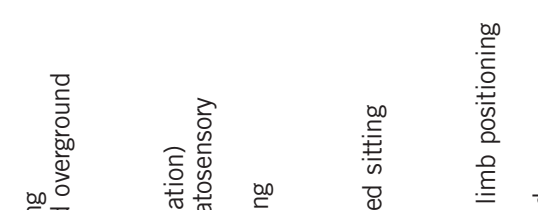

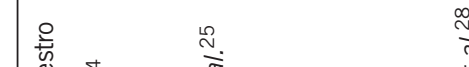

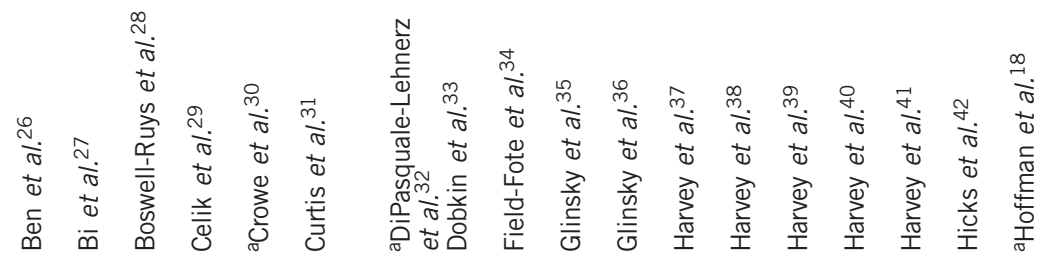

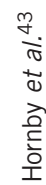




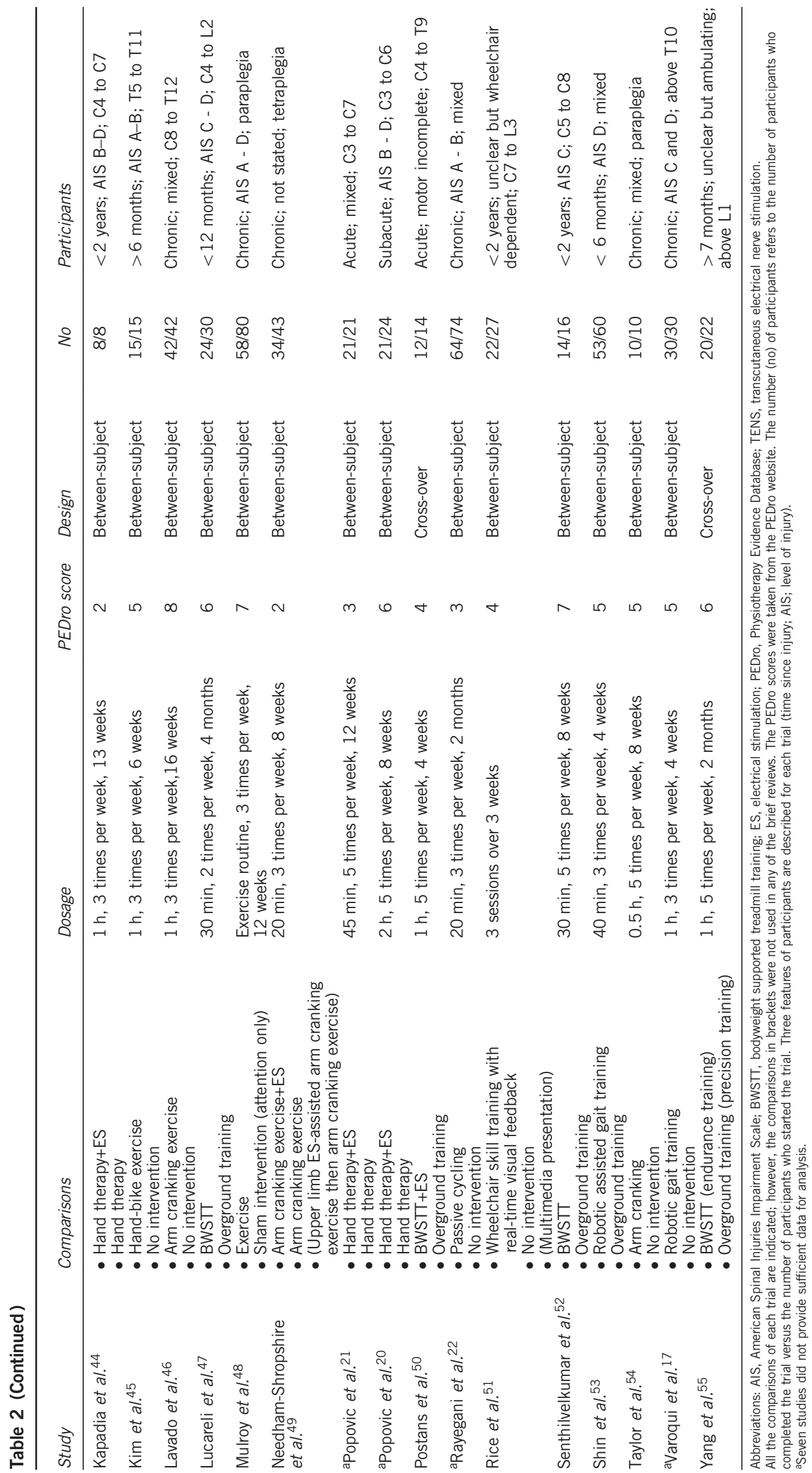


included an assessment at 6 weeks and 12 weeks, then only the data from the 6-week assessment were included.

We planned to deal with any type of data that may be extracted including time-to-event and count data; however, only continuous and dichotomous data were ultimately retrieved. The mean betweengroup differences $(95 \%$ confidence interval) and risk ratios were extracted, respectively. If these were not provided, then available post or change data were used to derive between group differences using the methodology recommended by Cochrane. ${ }^{16}$

\section{Methodological quality of the included trials}

The methodological quality of each trial was assessed using the PEDro scale (Table 2). The PEDro scale has 10 items that address key issues of bias. A total score of ten indicates minimal susceptibility to bias. The scores were attained from the PEDro website for all trials, except two, which were scored by the authors because they were not on the website. ${ }^{17,18}$ The scores on the PEDro website have been verified by two independent and formally trained raters from the Centre of Evidence-Based Physiotherapy.

\section{DATA SYNTHESIS}

\section{Statistical analysis}

Data from trials for each brief review (that is, for each combination of intervention and outcome) were pooled if possible using metaanalyses provided there was not statistical $\left(I^{2}>60 \%\right)$ or clinical heterogeneity. The 'metan' and 'admetan' commands of Stata (StataCorp, College Station, TX, USA) were used to generate forest plots and conduct all meta-analyses. Results were cross-checked using RevMan 5.1 software. A random-effects model was used for all meta-analyses. If continuous outcomes were similar across trials, then a weighted mean difference was calculated. If continuous outcomes measuring the same construct were different, then results were pooled using a standardised mean difference. A risk ratio was calculated for dichotomous data.

\section{Definition of treatment effectiveness}

The results of each brief review were defined as effective, ineffective or inconclusive according to the between group differences. The overall between-group difference was used for the brief reviews with a metaanalysis, and the between-group difference of individual trials was used for the brief reviews without a meta-analysis. The following decision rule was used:

1. Effective. An intervention was classified as effective if the lower end of the 95\% confidence interval (CI) of the between-group difference fell above the minimally worthwhile treatment effect.

2. Ineffective. An intervention was classified as ineffective if the upper end of the $95 \%$ CI of the between-group difference fell below the minimally worthwhile treatment effect.

3. Inconclusive. An intervention was classified as inconclusive if the 95\% CI of the between-group difference spanned the minimally worthwhile treatment effect.

The minimally worthwhile treatment effect was defined according to the value articulated by the authors of the original trial provided it was defined prior to the commencement of the trial. When a minimally worthwhile treatment effect was not articulated by authors a priori or when there was more than one trial in a brief review, the minimally worthwhile treatment effect was set as equivalent to $10 \%$ of mean post-values for the control groups. The minimally worthwhile treatment effect was set as 0.2 s.d. for brief reviews with meta-analyses expressed in standardised mean differences.

\section{Grading the strength of evidence}

The strength of evidence was only rated for brief reviews that indicated a treatment was either effective or ineffective. It was not rated for brief reviews with inconclusive findings. The Grading of Recommendations Assessment, Development and Evaluation (GRADE) methodology was used. ${ }^{19}$ GRADE uses a four-point scale (high quality, moderate quality, low quality and very low quality) based on a number of factors including the risk of bias in the trials, consistency of results across trials, the precision of estimates and the size of treatment effects. The PEDro scores for each trial were used to guide judgments about the risk of bias, although other potential sources of serious bias not captured by the PEDro scores were also considered.

\section{RESULTS}

Flow of studies through the review

The search retrieved 15784 papers. A total of 147 papers were randomised controlled trials involving people with SCI and were potentially eligible, but after evaluating the full text and excluding duplicate publications only 38 trials met the inclusion criteria (Table 2). ${ }^{17,18,20-55}$

\section{Description of the trials}

Seven trials did not provide sufficient data for analysis. ${ }^{17,18,20-22,30,32}$ The remaining 31 trials were relevant to 15 of the 22 brief reviews (Table 1). One trial had three arms and included two relevant comparisons. ${ }^{43}$ Another two trials had interventions and outcomes that were relevant to more than one of the brief reviews. ${ }^{42,48}$ Data from these trials were therefore used in more than one brief review. Meta-analysis was appropriate for 8 of the brief reviews.

\section{Findings}

Four reviews indicated that treatments were effective (Table 1 and Figure 1). The treatments were fitness training, wheelchair mobility training, hand training and TENS. These four reviews included 8 trials of 201 participants or limbs (for trials using within-subject designs). The GRADE strength of evidence for each of these reviews was either moderate or very low.

One review indicated that a treatment was ineffective (see Table 1 and Figure 2). The treatment was stretch for joint range of motion. This review included three trials of 100 participants or limbs (for trials using within-subject designs). The GRADE strength of evidence for this review was moderate.

The results of 10 reviews were inconclusive (Table 1 and Figure 3) - that is, they failed to rule in or rule out a possible therapeutic effect. The treatments included BWSTT (with or without ES) and robotic gait training compared with overground gait training, strength training (with or without ES), passive movements, seated mobility training and general exercise (for pain).

There were no trials with useable data relevant to seven reviews. The treatments included overground gait training, robotic gait training and BWSTT (with and without ES) compared with no or sham intervention, hand training with ES and cycling with ES.

\section{DISCUSSION}

Many papers, systematic reviews and clinical practice guidelines have summarised the evidence underpinning different physiotherapy interventions for people with SCI. However, our summary of the evidence is unique because of its wide scope and because it examines the effectiveness of commonly administered physiotherapy interventions in one paper. We defined a primary outcome for each review and we worked to a protocol. Our protocol was driven by clinical questions 
1. Fitness training vs no or sham intervention for fitness $(n=90)$

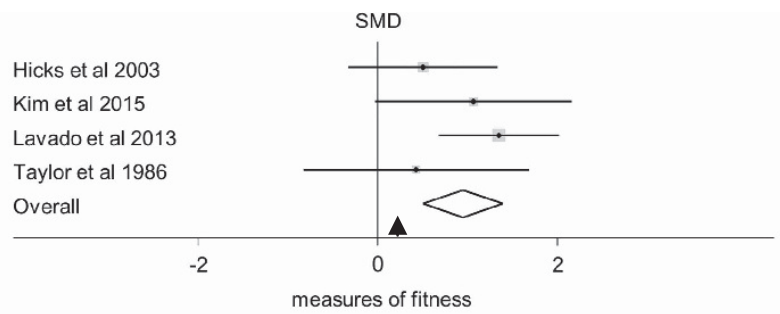

favours no intervention favours fitness training

3. Wheelchair mobility training vs no or sham intervention for wheelchair mobility $(n=18)$

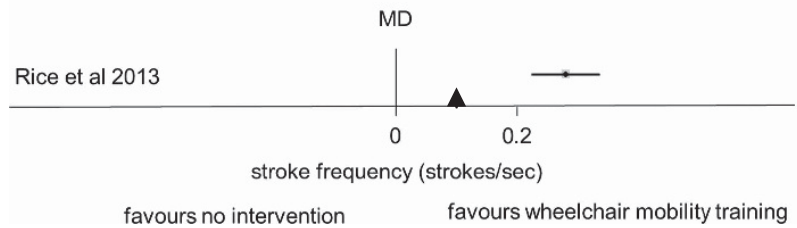

\section{TENS vs no or sham intervention for pain} $(\mathbf{n}=\mathbf{8 1})$

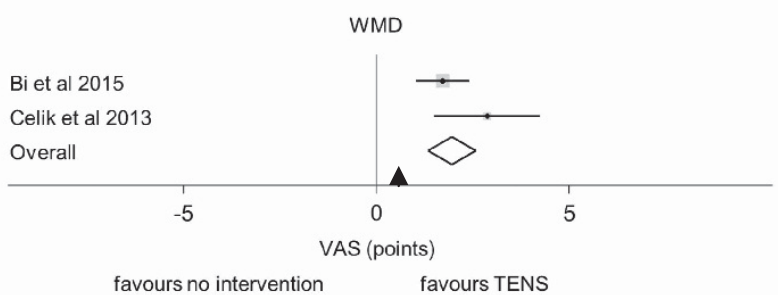

4. Hand training vs no or sham intervention for hand function $(n=12)$

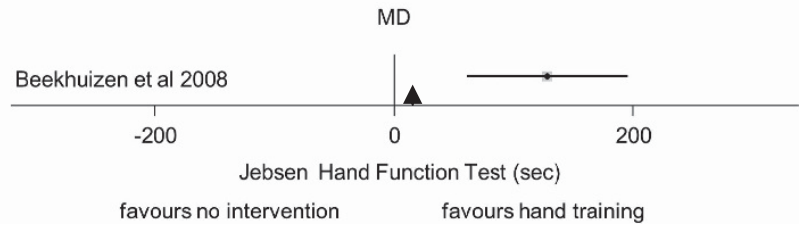

Figure 1 Forest plots summarising the results of the four brief reviews that indicate an intervention is clearly effective. Meta-analyses were appropriate for two of the reviews, and the conclusions of these reviews are based on the overall between-group differences. Meta-analyses were not possible for the other two brief reviews, and conclusions are based on the between-group differences of the individual trials. The results are expressed as mean differences (MDs), weighted mean differences (WMDs) or standardised mean differences (SMDs). The minimally worthwhile treatment effect is indicated by the symbol ( $\mathbf{A}$ ) on the $x$ axis. The number of participants or limbs (for trials using within-subject designs) contributing to the analyses is indicated.

expressed in PICO format where $\mathrm{P}$ reflects participants, I reflects intervention, $\mathrm{C}$ reflects comparison and $\mathrm{O}$ reflects outcome. In addition, we interpreted our results with respect to a pre-defined minimally worthwhile treatment effect for each brief review. This approach minimises the risk of spurious findings and conclusions.

The results of our brief reviews indicate evidence to support four interventions; however, the strength of evidence is not high for any of these interventions and only moderate for two of them (i.e., fitness training and TENS). The results of the remaining brief reviews are either inconclusive or in the case of stretch indicate that the treatment is ineffective. Interestingly, there were no trials with usable data for 7 of the 22 brief reviews. Importantly, lack of evidence does not mean that interventions are ineffective. Lack of evidence does, however, justify reconsidering our confidence about the effectiveness of some widely accepted interventions and should prompt us to question some long-held assumptions about what physiotherapists should and should not do. The failure of physiotherapy research to demonstrate treatment effectiveness is not unique to physiotherapy and SCI, nor is it unique to rehabilitation.

Other summaries of evidence include non-randomised trials and soft evidence. Some argue that we need to revert to this type of evidence because of the paucity of randomised controlled trials. However, this type of evidence is highly vulnerable to different sources of bias that tend to exaggerate treatment effectiveness. It therefore gives a distorted impression of the real situation and may only serve to give misplaced confidence about the efficacy of different interventions. This type of evidence is particularly vulnerable to publication bias because non-randomised trials and soft evidence are unlikely to be published if the results are negative.

The interpretation of each brief review relies on our definitions of minimally worthwhile treatment effects. Our use of minimally worthwhile treatment effects enabled us to consider the size of treatment effects and distinguish between results that are inconclusive and results that provide evidence that a treatment is ineffective. Some

\section{Stretch vs no or sham intervention for joint range of motion $(n=100)$}

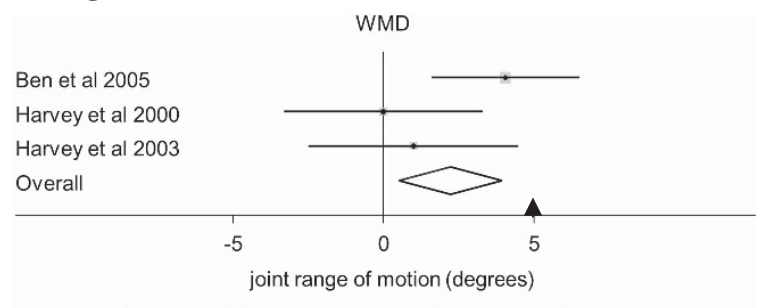

favours no intervention

favours stretch

Figure 2 Forest plots summarising the results of the one brief review that indicates an intervention is clearly ineffective. The conclusions of this review are based on the overall between-group differences. The results are expressed as a weighted mean difference (WMD). The minimally worthwhile treatment effect is indicated by the symbol $(\boldsymbol{\Lambda})$ on the $x$ axis. The number of participants or limbs (for trials using within-subject designs) contributing to the analyses is indicated.

may disagree with our definitions of minimally worthwhile treatment effects, and this may slightly change the conclusions of some reviews. The review most likely to be affected by a change in the definition of its minimally worthwhile treatment effect is the review comparing BWSTT with overground gait training. We concluded that it is not clear whether BWSTT is superior to overground gait training on the basis of how fast control participants of the included studies walked. However, regardless, some physiotherapists and patients may want to see an added treatment benefit of at least $0.1 \mathrm{~m} / \mathrm{sec}$ in gait velocity to justify the use of BWSTT. If this is the case, then the results of our brief review indicate that BWSTT is not superior to overground gait training. Clearly, clinicians and patients need to make their own decisions about minimally worthwhile treatment effects and then interpret the results of each brief review accordingly.

The findings of all the brief reviews need to be interpreted in the context of the comparisons. For example, the failure to demonstrate 
that stretch applied by a physiotherapist is ineffective does not mean that stretch as typically incorporated into routine care is also ineffective. Clinical trials can only answer questions about the relative effectiveness of the two interventions examined in the trial. Dosage is also clearly a critical aspect of a trial, and the failure of some trials to demonstrate treatment effectiveness may reflect insufficient treatment dosages. For example, perhaps strengthening and stretching exercises need to be applied at much higher dosages than typically applied in clinical trials and perhaps for many months or even years.

The 22 selected interventions reflect those most widely administered in clinical practice. They were chosen on the basis of studies that have systematically quantified the types of interventions commonly administered by physiotherapists ${ }^{56-60}$ and on the basis of the

\section{BWSTT vs overground training for gait}

$$
(\mathrm{n}=\mathbf{2 1 8})
$$
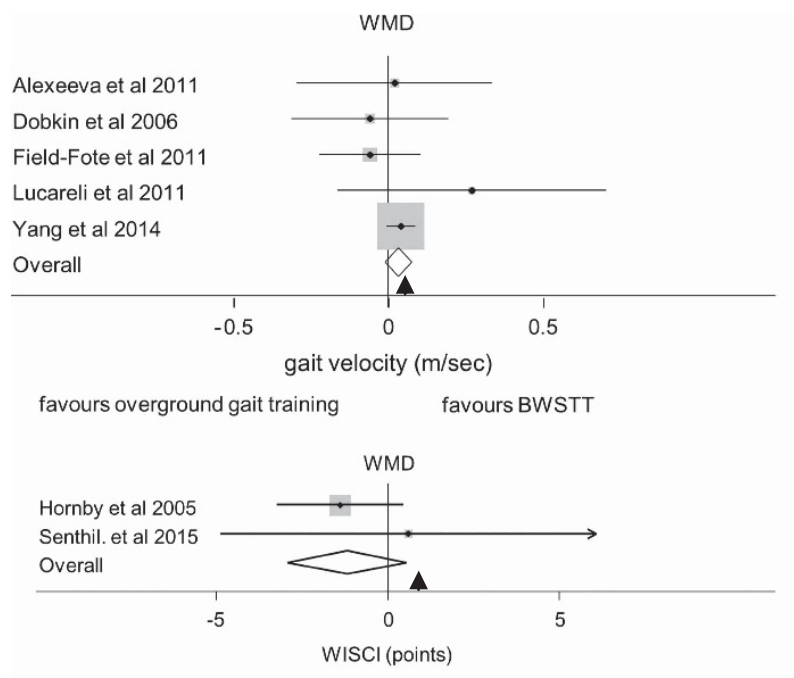

favours overground gait training favours BWSTT

\section{BWSTT + ES vs overground training for gait $(\mathrm{n}=16)$}

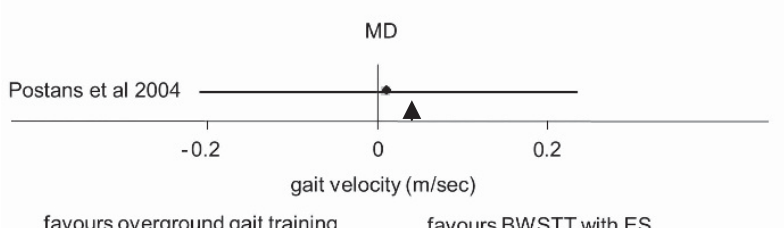

5. Passive movements vs no or sham intervention for joint range of motion $(n=40)$

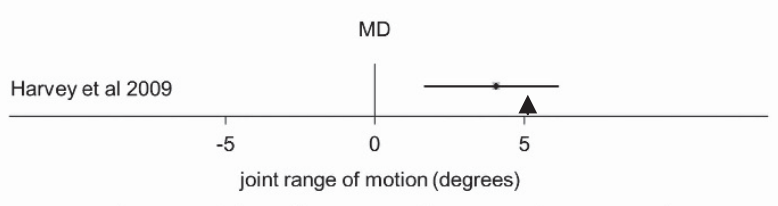

favours no intervention

favours passive movements physiotherapy module of www.elearnSCI.org. Of course some may disagree with our choice of the 22 most widely administered interventions and the primary outcomes that we selected to reflect their effectiveness. Future studies could use a Delphi process to get consensus among physiotherapists around the world to clarify these issues or repeat existing observational studies that have attempted to clarify the most widely used physiotherapy interventions on a larger sample of SCI units from many different countries. Interestingly the majority of research attention is being directed at BWSTT and robotic gait training with comparatively little research attention being directed at some of the more mundane but widely administered interventions such as strengthening and stretching exercises. ${ }^{56-60}$

\section{Robotic gait training vs overground training for gait $(\mathrm{n}=\mathbf{1 3 1})$}

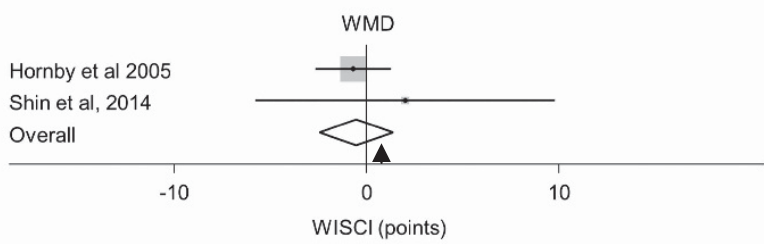

favours overground gait training favours robotic gait training

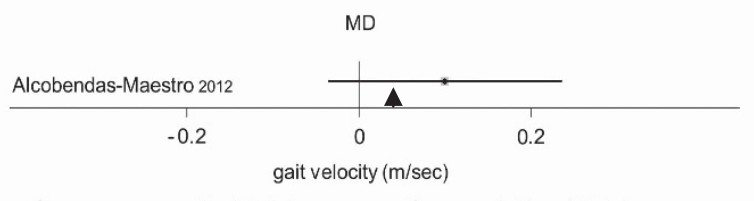

favours overground gait training favours robotic gait training

4. Strength training for non-paralysed muscle vs no or sham intervention for voluntary strength $(\mathbf{n}=\mathbf{8 1})$

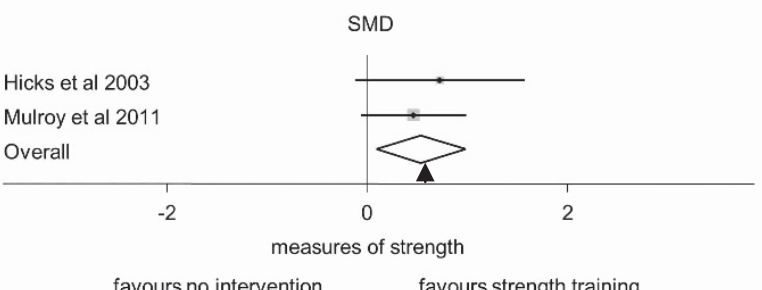

6. Seated mobility training vs no or sham intervention for seated mobility $(n=62)$

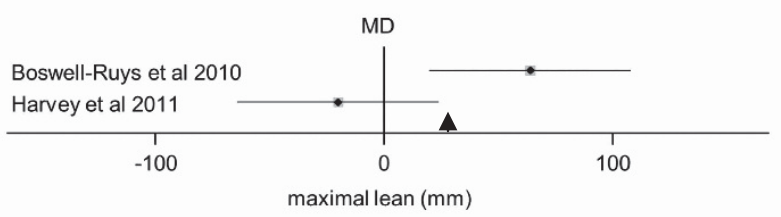

favours no intervention favours seated mobility training

Figure 3 Forest plots summarising the results of the 10 brief reviews with inconclusive findings. Meta-analyses were appropriate for five of the reviews, and the conclusions of these reviews are based on the overall between-group differences. Meta-analyses were either not appropriate or not possible for the other five brief reviews, and conclusions are based on the between-group differences of the individual trials. The results are expressed as mean differences (MDs), weighted mean differences (WMDs), standardised mean differences (SMDs) or risk ratio (RR). The minimally worthwhile treatment effect is indicated by the symbol ( $\boldsymbol{\Delta}$ ) on the $x$ axis. The number of participants or limbs (for trials using within-subject designs) contributing to the analyses is indicated. 
7. Strength training for partially-paralysed

muscles vs no or sham intervention for

voluntary strength $(\mathbf{n}=\mathbf{3 1})$

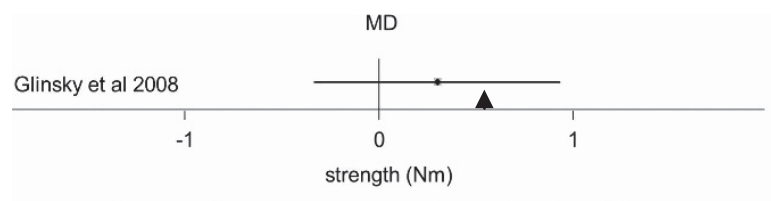

favours no intervention favours strength training

9. General exercise vs no or sham intervention

for pain $(n=116)$

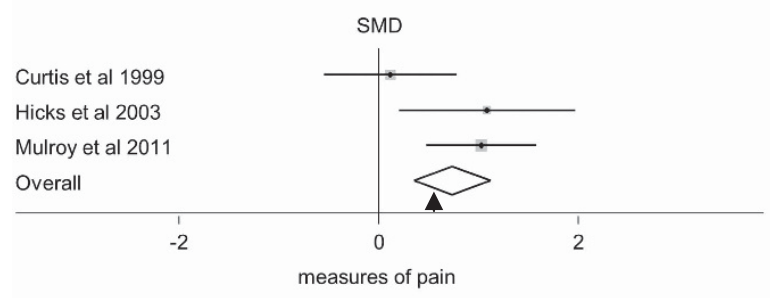

favours no intervention favours general exercise
8. Strength training and ES for partially-

paralysed muscles vs no or sham intervention

for voluntary strength $(n=20)$

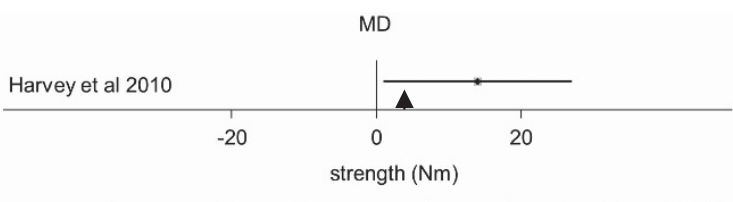

favours no intervention favours strength training with ES

10. ES for partially-paralysed muscles vs no or sham intervention for voluntary strength $(\mathrm{n}=111)$

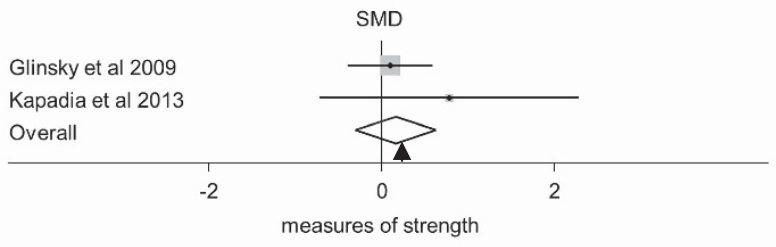

favours no intervention favours electrical stimulation

RR

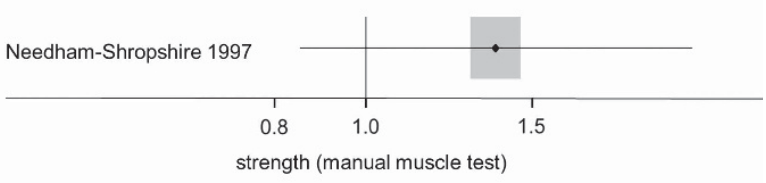

favours no intervention

favours electrical stimulation

Figure 3 Continued.

There are three main limitations of this systematic review. First, we may have introduced bias when selecting the relevant outcomes from each trial. We think that this is unlikely because as far as possible we made decisions about the choice of outcomes prior to examining the results of trials. Second, we did not include trials that compared different types of interventions (except for BWSTT and robotic gait training, which were compared with overground gait training). We restricted our inclusion criteria in this way to keep the review manageable but also to restrict conclusions to the effectiveness of interventions per se. The relative effectiveness of different interventions is a more complex question. It becomes particularly complex when results fail to demonstrate that one treatment is superior to another because without a control group it is not known whether both treatments are effective or both treatments are ineffective. Thus, as a first step to summarising the evidence, it is important to examine the effectiveness of interventions compared with no intervention or sham interventions (or usual care provided both groups received usual care). The third limitation of this study is that we only looked at the effectiveness of each intervention on one outcome. We selected each outcome for each intervention a priori and on the basis of the most common reason why an intervention is administered by physiotherapists. For example, BWSTT is most widely used to improve gait. Hence, for this intervention, the outcome of interest was gait. However, BWSTT may also have other therapeutic benefits that were not captured.

This systematic review provides an overview of the existing evidence related to common questions about the effectiveness of different physiotherapy interventions for people with SCI. It indicates initial evidence for four interventions. However, there are a lot of uncertainties about most of the widely used physiotherapy interventions for people with SCI. Without a strong evidence base for current clinical practice, all new and innovative interventions and all trials designed to compare different interventions are building on shifting and possibly incorrect assumptions about the effectiveness or ineffectiveness of current physiotherapy treatments. Therefore, future research needs to not only explore new interventions but also build a strong evidence base to current practice. A strong evidence base relies on research that is void of bias.

\section{CONFLICT OF INTEREST}

The authors declare no conflict of interest.

1 Eng JJ, Teasell R, Miller WC, Wolfe DL, Townson AF, Aubut JA et al. The Spinal Cord Injury Rehabilitation Evidence: Methods of the SCIRE Systematic Review. Top Spinal Cord Inj Rehabil 2007; 13: 1-10.

2 Lu X, Battistuzzo CR, Zoghi M, Galea MP. Effects of training on upper limb function after cervical spinal cord injury: a systematic review. Clin Rehabil 2015; 29: 3-13.

3 Morawietz C, Moffat F. Effects of locomotor training after incomplete spinal cord injury: A systematic review. Arch Phys Med Rehabil 2013; 94: 2297-2308.

4 Mehta S, Orenczuk K, McIntyre A, Willems G, Wolfe DL, Hsieh JT et al. Neuropathic pain post spinal cord injury part 1: systematic review of physical and behavioral treatment. Top Spinal Cord Inj Rehabil 2013; 19: 61-77.

5 Hicks AL, Martin Ginis KA, Pelletier CA, Ditor DS, Foulon B, Wolfe DL. The effects of exercise training on physical capacity, strength, body composition and functional performance among adults with spinal cord injury: a systematic review. Spinal Cord 2011; 49: 1103-1127.

6 Spooren Al, Janssen-Potten YJ, Kerckhofs E, Seelen HA. Outcome of motor training programmes on arm and hand functioning in patients with cervical spinal cord injury 
according to different levels of the ICF: a systematic review. J Rehabil Med 2009; 41: 497-505.

7 Harvey L, Lin CM, Glinsky J, De Wolf A. The effectiveness of physical interventions for people with spinal cord injuries: a systematic review. Spinal Cord 2009; 47: 184-195.

8 Valent L, Dallmeijer A, Houdijk H, Talsma E, van der Woude L. The effects of upper body exercise on the physical capacity of people with a spinal cord injury: a systematic review. Clin Rehabil 2007; 21: 315-330.

9 Lam T, Eng JE, Wolfe DL, Hsieh JTC, Whittaker M. A systematic review of the efficacy of gait rehabilitation strategies for spinal cord injury. Top Spinal Cord Inj Rehabil 2007; 13: 32-57.

10 Wessels M, Lucas C, Eriks I, de Groot S. Body weight-supported gait training for restoration of walking in people with an incomplete spinal cord injury: a systematic review. J Rehabil Med 42: 513-519.

11 Mehrholz J, Kugler J, Pohl M. Locomotor training for walking after spinal cord injury. Cochrane Database Syst Rev 2012; 11: CD006676.

12 Ginis KAM, Hicks AL, Latimer AE, Warburton DER, Bourne C, Ditor DS et al. The development of evidence-informed physical activity guidelines for adults with spinal cord injury. Spinal Cord 2011; 49: 1088-1096.

13 Boldt I, Eriks-Hoogland I, Brinkhof MW, de Bie R, Joggi D, von Elm E. Nonpharmacological interventions for chronic pain in people with spinal cord injury. Cochrane Database Syst Rev 2014; 11: Cd009177.

14 Katalinic OM, Harvey LA, Herbert RD, Moseley AN, Lannin NA, Schurr K. Stretch for the treatment and prevention of contractures. Cochrane Database Syst Rev 2010, CD007455.

15 Prabhu R, Swaminathan N, Harvey L. Passive movements for the treatment and prevention of contractures. Cochrane Database Syst Rev 2013; 12: CD009331.

16 Higgins J, Green S. (eds). Cochrane Handbook for Systematic Reviews of Interventions Version 5.1.0 [updated March 2011]. The Cochrane Collaboration, 2011. Available from www.cochrane-handbook.org 2011.

17 Varoqui D, Niu X, Mirbagheri MM. Ankle voluntary movement enhancement following robotic-assisted locomotor training in spinal cord injury. J Neuroeng Rehabil 2014; 11 46.

18 Hoffman L, Field-Fote E. Effects of practice combined with somatosensory or motor stimulation on hand function in persons with spinal Cord Injury. Top Spinal Cord Inj Rehabil 2013; 19: 288-299.

19 Guyatt GH, Oxman AD, Vist GE, Kunz R, Falck-Ytter Y, Alonso-Coello P et al. GRADE: an emerging consensus on rating quality of evidence and strength of recommendations. Br Med J 2008; 336: 924-926.

20 Popovic MR, Kapadia N, Zivanovic V, Furlan JC, Craven BC, McGillivray C. Functional electrical stimulation therapy of voluntary grasping versus only conventional rehabilitation for patients with subacute incomplete tetraplegia: a randomized clinical trial. Neurorehabil Neural Repair 2011; 25: 433-442.

21 Popovic MR, Thrasher TA, Adams ME, Takes V, Zivanovic V, Tonack MI. Functional electrical therapy: retraining grasping in spinal cord injury. Spinal Cord 2006; 44: 143-151.

22 Rayegani SM, Shojaee H, Sedighipour L, Soroush MR, Baghbani M, Amirani OB. The effect of electrical passive cycling on spasticity in war veterans with spinal cord injury. Front Neurol 2011; 2: 39.

23 Alcobendas-Maestro M, Esclarin-Ruz A, Casado-Lopez RM, Munoz-Gonzalez A, Perez-Mateos G, Gonzalez-Valdizan E et al. Lokomat robotic-assisted versus overground training within 3 to 6 months of incomplete spinal cord lesion: randomized controlled trial. Neurorehabil Neural Repair 2012; 26: 1058-1063.

24 Alexeeva N, Sames C, Jacobs PL, Hobday L, Distasio MM, Mitchell SA et al. Comparison of training methods to improve walking in persons with chronic spinal cord injury: a randomized clinical trial. J Spinal Cord Med 2011; 34: 362-379.

25 Beekhuizen KS, Field-Fote EC. Sensory stimulation augments the effects of massed practice training in persons with tetraplegia. Arch Phys Med Rehabil 2008; 89: 602-608.

26 Ben M, Harvey L, Denis S, Glinsky J, Goehl G, Chee S et al. Does 12 weeks of regular standing prevent loss of ankle mobility and bone mineral density in people with recent spinal cord injuries? Aust J Physiother 2005; 51: 251-256.

$27 \mathrm{Bi} X$, Lv H, Chen B-L, Li X, Wang X-Q. Effects of transcutaneous electrical nerve stimulation on pain in patients with spinal cord injury: a randomized controlled trial. J Phys Ther Sci 2015; 27: 23-25.

28 Boswell-Ruys CL, Harvey LA, Barker JJ, Ben M, Middleton JW, Lord SR. Training unsupported sitting in people with chronic spinal cord injuries: a randomized controlled trial. Spinal Cord 2010; 48: 138-143.

29 Celik EC, Erhan B, Gunduz B, Lakse E. The effect of low-frequency TENS in the treatment of neuropathic pain in patients with spinal cord injury. Spinal Cord 2013; 51: 334-337.

30 Crowe J, MacKay-Lyons M, Morris H. A multi-centre, randomized controlled trial of the effectiveness of positioning on quadriplegic shoulder pain. Physiother Can 2000; 52: 266-273.

31 Curtis KA, Tyner TM, Zachary L, Lentell G, Brink D, Didyk T et al. Effect of a standard exercise protocol on shoulder pain in long-term wheelchair users. Spinal Cord 1999; 37: 421-429.

32 DiPasquale-Lehnerz P. Orthotic intervention for development of hand function with C-6 quadriplegia. Am J Occup Ther 1994; 48: 138-144.

33 Dobkin B, Apple D, Barbeau H, Basso M, Behrman A, Deforge D et al. Weight-supported treadmill vs over-ground training for walking after acute incomplete SCl. Neurology 2006; 66: 484-493.
34 Field-Fote EC, Roach KE. Influence of a locomotor training approach on walking speed and distance in people with chronic spinal cord injury: a randomized clinical trial. Phys Ther 2011; 91: 48-60.

35 Glinsky J, Harvey L, Korten M, Drury C, Chee S, Gandevia SC. Short-term progressive resistance exercise may not be effective for increasing wrist strength in people with tetraplegia: a randomised controlled trial. Aust J Physiother 2008; 54: 103-108.

36 Glinsky J, Harvey L, van Es P, Chee S, Gandevia SC. The addition of electrical stimulation to progressive resistance training does not enhance the wrist strength of people with tetraplegia: a randomized controlled trial. Clin Rehabil 2009; 23: 696-704.

37 Harvey LA, Batty J, Crosbie J, Poulter S, Herbert RD. A randomized trial assessing the effects of 4 weeks of daily stretching on ankle mobility in patients with spinal cord injuries. Arch Phys Med Rehabil 2000; 81: 1340-1347.

38 Harvey LA, Byak AJ, Ostrovskaya M, Glinsky J, Katte L, Herbert RD. Randomised trial of the effects of four weeks of daily stretch on extensibility of hamstring muscles in people with spinal cord injuries. Aust J Physiother 2003; 49: 176-181.

39 Harvey LA, Herbert RD, Glinsky J, Moseley AM, Bowden J. Effects of 6 months of regular passive movements on ankle joint mobility in people with spinal cord injury: a randomized controlled trial. Spinal Cord 2009; 47: 62-66.

40 Harvey LA, Fornusek C, Bowden JL, Pontifex N, Glinsky J, Middleton JW et al. Electrical stimulation plus progressive resistance training for leg strength in spinal cord injury: A randomized controlled trial. Spinal Cord 2010; 48: 570-575.

41 Harvey L, Ristev D, Hossain M, Hossain M, Bowden J, Boswell-Ruys C et al. Training unsupported sitting does not improve ability to sit in people with recently-acquired paraplegia: a randomised controlled trial. J Physiother 2011; 57: 83-90.

42 Hicks AL, Martin KA, Ditor DS, Latimer AE, Craven C, Bugaresti J et al. Long-term exercise training in persons with spinal cord injury: effects on strength, arm ergometry performance and psychological well-being. Spinal Cord 2003; 41: 34-43.

43 Hornby TG, Campbell DD, Zemon DH, Kahn JH. Clinical and quantitative evaluation of robotic-assisted treadmill walking to retrain ambulation after spinal cord injury. Top Spinal Cord Inj Rehabil 2005; 11: 1-17.

44 Kapadia N, Zivanovic V, Popovic MR. Restoring voluntary grasping function in individuals with incomplete chronic spinal cord injury: Pilot Study. Top Spinal Cord Inj Rehabil 2013; 19: 279-287.

$45 \mathrm{Kim}$ DI, Lee H, Lee BS, Kim J, Jeon JY. Effects of a six-week indoor hand-bike exercise program on health and fitness levels in people with spinal cord injury: a randomized controlled trial study. Arch Phys Med Rehabil 2015; 96: 2033-2040.

46 Lavado EL, Cardoso JR, Silva LG, Dela Bela LF, Atallah AN. Effectiveness of aerobic physical training for treatment of chronic asymptomatic bacteriuria in subjects with spinal cord injury: a randomized controlled trial. Clin Rehabil 2013; 27: 142-149.

47 Lucareli PR, Lima MO, Lima FPS, De Almeida JG, Brech GC, D'Andréa Greve JM. Gait analysis following treadmill training with body weight support versus conventional physical therapy: A prospective randomized controlled single blind study. Spinal Cord 2011; 49: 1001-1007.

48 Mulroy SJ, Thompson L, Kemp B, Hatchett PP, Newsam CJ, Lupold DG et al. Strengthening and optimal movements for painful shoulders (STOMPS) in chronic spinal cord injury: A randomized controlled trial. Phys Ther 2011; 91: 305-324.

49 Needham-Shropshire BM, Broton JG, Cameron TL, Klose KJ. Improved motor function in tetraplegics following neuromuscular stimulation-assisted arm ergometry. J Spinal Cord Med 1997; 20: 49-55.

50 Postans NJ, Hasler JP, Granat MH, Maxwell DJ. Functional electrical stimulation to augment partial weight-bearing supported treadmill training for patients with acute incomplete spinal cord injury: A pilot study. Arch Phys Med Rehabil 2004; 85: 604-610.

51 Rice IM, Pohlig RT, Gallagher JD, Boninger ML. Hand rim wheelchair propulsion training effect on over ground propulsion using biomechanical real time visual feedback. Arch Phys Med Rehabil 2013; 94: 256-263.

52 Senthilvelkumar T, Magimairaj H, Fletcher J, Tharion G, George J. Comparison of body weight-supported treadmill training versus body weight-supported overground training in people with incomplete tetraplegia: a pilot randomized trial. Clin Rehabil 2015; 29: 42-49.

53 Shin JC, Kim JY, Park HK, Kim NY. Effect of robotic-assisted gait training in patients with incomplete spinal cord injury. Ann Rehabil Med 2014; 38: 719-725.

54 Taylor AW, McDonell E, Brassard L. The effects of an arm ergometer training programme on wheelchair subjects. Paraplegia 1986; 24: 105-114.

55 Yang JF, Musselman KE, Livingstone D, Brunton K, Hendricks G, Hill D et al. Repetitive mass practice or focused precise practice for retraining walking after incomplete spinal cord injury? A pilot randomized clinical trial. Neurorehabil Neural Repair 2014; 28 : 314-324.

56 Van Langeveld SA, Post MW, Van Asbeck FW, Ter Horst P, Leenders J, Postma K et al. Contents of physical therapy, occupational therapy, and sports therapy sessions for patients with a spinal cord injury in three Dutch rehabilitation centres. Disabil Rehabil 2011; 33: 412-422.

57 Zanca JM, Natale A, Labarbera J, Schroeder ST, Gassaway J, Backus D. Group physical therapy during inpatient rehabilitation for acute spinal cord injury: findings from the SCIRehab Study. Phys Ther 2011; 91: 1877-1891.

58 Teeter L, Gassaway J, Taylor S, LaBarbera J, McDowell S, Backus D et al. Relationship of physical therapy inpatient rehabilitation interventions and patient characteristics to outcomes following spinal cord injury: The SCIRehab project. J Spinal Cord Med 2012; 35: 503-526. 
59 Natale A, Taylor S, LaBarbera J, Bensimon L, McDowell S, Mumma SL et al. SCIRehab project series: The physical therapy taxonomy. J Spinal Cord Med 2009; 32 : 270-282.
60 Whiteneck G, Gassaway J, Dijkers M, Jha A. New approach to study the contents and outcomes of spinal cord injury rehabilitation: The SCIRehab project. J Spinal Cord Med 2009; 32: 251-259. 